Research Paper

\title{
Multi-Parametric MRI-Directed Focal Salvage Perma- nent Interstitial Brachytherapy for Locally Recurrent Adenocarcinoma of the Prostate: A Novel Approach
}

\author{
T. Wallace ${ }^{1,2,5^{凶}}$, I. Avital ${ }^{1,3 凶}$, A. Stojadinovic ${ }^{4}$, B. Brucher ${ }^{5}$, E. Coté ${ }^{6}$ and J. Yu ${ }^{7}$ \\ 1. Bon Secours Cancer Institute, Bon Secours Health Care System, Richmond VA, USA. \\ 2. Division of Radiation Oncology, Bon Secours Health Care System, Richmond VA, USA. \\ 3. Division of Surgical Oncology, Bon Secours Health Care System, Richmond VA, USA. \\ 4. Department of Surgery, Division of Surgical Oncology, Walter Reed National Military Medical Center, Bethesda, MD, USA. \\ 5. Theodor-Billroth-Academy®, Josephsburgstrasse 6, 81673 Munich, Germany. \\ 6. Virginia Urology, Richmond VA, USA. \\ 7. Virginia Commonwealth University Health System, Richmond VA, USA.
}

$\triangle$ Corresponding author: Timothy Wallace, M.D., Ph.D. Division of Radiation Oncology, Bon Secours Cancer Institute (BSCI),Richmond, VA 23230 USA. Phone: (804) 266-7762 Fax: (804) 266-0344 E-mail: timothywallace1@gmail.com Or Itzhak Avital, MD. Medical Director, Bon Secours Cancer Institute (BSCI), Richmond, VA 23230, USA. Email: Itzhak_avital@bshsi.org.

( ) Ivyspring International Publisher. This is an open-access article distributed under the terms of the Creative Commons License (http://creativecommons.org/ licenses/by-nc-nd/3.0/). Reproduction is permitted for personal, noncommercial use, provided that the article is in whole, unmodified, and properly cited.

Received: 2012.10.19; Accepted: 2013.01.15; Published: 2013.02.01

\begin{abstract}
Even with the technological advances of dose-escalated IMRT with the addition of the latest image guidance technologies, local failures still occur. The combination of MRI-based imaging techniques can yield quantitative information that reflects on the biological properties of prostatic tissues. These techniques provide unique information that can be used for tumor detection in the treated gland. With the advent of these improved imaging modalities, it has become possible to more effectively image local recurrences within the prostate gland. With better imaging, these focal recurrences can be differentially targeted with salvage brachytherapy minimizing rectal and bladder toxicity. Here we report a novel use of MRI-directed focal brachytherapy after local recurrence. This technique offers a unique opportunity to safely and successfully treat recurrent prostate cancer, previously treated with definitive radiation therapy. The use of multi-parametric MRI-directed focal salvage permanent interstitial brachytherapy for locally recurrent adenocarcinoma of the prostate is a promising strategy to avoid more aggressive and expensive treatments that are associated with increased morbidity, potentially improving survival at potentially lower costs.
\end{abstract}

Key words: Prostate cancer, Salvage therapy, Brachytherapy Multi-parametric MRI.

\section{Introduction}

There currently is no standard salvage treatment for locally recurrent adenocarcinoma of the prostate $(\mathrm{CaP})$ after definitive external beam radiation treatment (EBRT). Patients with recurrent $\mathrm{CaP}$ are often given androgen deprivation therapy, which is a temporizing measure, at best, and is rarely curative. Salvage prostatectomy is associated with 10 year bio- chemical recurrence-free survival of less than $50 \%$ (1) and is associated with a significant rate of post-operative incontinence ( $21 \%$ to $90 \%)$, erectile dysfunction $(80 \%$ to $100 \%)$, bladder neck contractures ( $22 \%$ to $41 \%)$, and rectal injury ( $0 \%$ to $28 \%)(2,3,4)$. Salvage cryotherapy is associated with 5 -year biochemical control rates of $<50 \%$, with attendant uri- 
nary incontinence rates of $73 \%$, obstructive uropathy symptoms of $67 \%$ and impotence rates of $72 \%$ (5). Salvage brachytherapy $\left(\right.$ Brachy $\left._{\mathrm{x}}\right)$ has been associated with biochemical control rates between $34 \%$ and $54 \%(6,7)$. In one phase II prospective randomized trial of magnetic resonance imaging (MRI)-guided salvage Brachy $\mathrm{R}_{\mathrm{x}}$ for local CaP recurrence after definitive radiation therapy, a 70\% 4-year PSA (Prostate-Specific Antigen) failure-free survival rate was observed and $28 \%$ of the patients experienced grade III or grade IV GU (Genitourinary) and/or GI (Gastrointestinal)toxicities.

Newer multi-parametric prostate MRI techniques which include T2, diffusion- weighted, dynamic contrast enhanced imaging and MR spectroscopy are allowing us to more accurately identify locally recurrent CaP after initial EBRT $(9,10,11)$. These newer MRI techniques have been used to guide focal salvage high dose rate Brachy $R_{x}$ for locally recurrent disease after primary EBRT (12) in addition to salvage permanent prostate Brachy $_{x}$ for locally recurrent disease after primary Brachy $R_{x}(13)$.

Herein we report the application of multi-parametric-MRI-(MPM) guided permanent interstitial prostate Brachy $R_{\mathrm{x}}$. This therapeutic modality was applied for locally recurrent prostate cancer after failure of primary EBRT. MPM-guided Brachy $R_{x}$ for focal salvage of recurrent $\mathrm{CaP}$ represents a novel treatment approach.

\section{Potential typical application of MPM in interstitially recurrent prostate cancer af- ter EBRT}

On July 18, 2007, a 68 year old Caucasian male was diagnosed with adenocarcinoma of the prostate. At presentation, he was found to have a palpable nodule at the right apex of his prostate on routine digital rectal exam. His pre-treatment PSA was abnormally elevated, $3.3 \mathrm{ng} / \mathrm{dl}$. He underwent a trans-rectal ultrasound (TRUS)-guided biopsy of the prostate nodule. Final pathology revealed adenocarcinoma with a Gleason score of $3+4$ in $20 \%$ of the core from the left mid gland medially and Gleason score $3+3$ in $20 \%$ of the core from the left apex. His prostate volume measured $31.14 \mathrm{ml}$ and his clinical stage of $\mathrm{CaP}$ was T2a.

Between October 18, 2007 and December 18, 2007, he received definitive 7-field IMRT (Intensity Modulated Radiation Therapy) to the prostate and proximal seminal vesicles to a total dose of 7,560 cGy delivered in 42 daily fractions at 180 cGy per fraction. This treatment was accompanied by Grade 1 urinary symptoms and Grade 2 proctitis; otherwise he tolerated his treatment well. His PSA nadir was at 0.19 ng/dl on February 4, 2009.
Table 1 shows the PSA values between 2009 and 2011. His calculated PSA doubling time for the same period was 8.49 months and his PSA velocity was 0.62 $\mathrm{ng} / \mathrm{ml} / \mathrm{yr}$. Bone scan on May 9, 2011 was negative for metastatic disease. Re-staging computed tomography (CT) and chest x-ray were negative for metastatic disease. On June 16, 2011, he underwent a repeat TRUS-guided biopsy of the prostate. Final pathology revealed recurrent adenocarcinoma with a Gleason score of $3+4$ involving $5 \%-10 \%$ of the core from the left mid gland medially and $3+4$ in $35 \%$ of the core from the left mid gland laterally. Digital rectal exam at the time of recurrence revealed a diffusely firm prostate without discrete nodularity. Bone scan on May 9, 2011 was negative for metastatic disease.

Table I. PSA values.

\begin{tabular}{ll}
\hline Date & PSA $(\mathrm{ng} / \mathrm{dl})$ \\
\hline $02 / 04 / 2009$ & 0.19 \\
$08 / 10 / 2009$ & 0.24 \\
$02 / 11 / 2010$ & 0.62 \\
$09 / 23 / 2010$ & 0.86 \\
$01 / 24 / 2011$ & 1.1 \\
$4 / 28 / 2011$ & 1.7 \\
\hline
\end{tabular}

He was sent for a multi-parametric prostate MRI on July 26, 2011 (Fig 1). A body coil and endorectal coil were utilized for this study on a $1.5 \mathrm{~T}$ magnet. Axial T2, sagittal T2 and coronal T2-weighted images, diffusion weighted images with b50, 400 and 1000, and dynamic contrast enhanced images were obtained through the prostate. A total of $20 \mathrm{ml}$ of ProHance was administered intravenously. The MRI findings revealed a vague low T2-weighted focus in the left mid gland measuring about $8.6 \mathrm{~mm} \times 11 \mathrm{~mm}$ in size. This low T2 signal focus was noted to extend to the prostate capsule without clear evidence of extracapsular extension of disease. There was correlating diffusion restriction in this region on b1000 image (Fig 1B). In addition, on the dynamic contrast-enhanced images, there was also a corresponding early enhancing focus with rapid washout (Fig 1C), correlating with the region at the left mid gland of the peripheral zone. This lesion corresponds to the region positive for recurrent disease on the most recent biopsy. In addition, there was generalized decreased T2 signal of the prostate consistent with prior radiation treatment, but there were no other focal abnormalities. Both seminal vesicles were unremarkable and there was no pelvic lymphadenopathy observed on T1-weighted images.

On September 8, 2011, the patient was taken to the operating room for salvage interstitial brachytherapy implant. The planning target volume (PTV) 
was defined as the MRI-positive disease plus $3 \mathrm{~mm}$. Palladium-103 seeds were implanted to a total dose of 124 Gy. The intra-operative D90 was $100 \%$ and the V100 was $100 \%$. The rectal V100 was $0.00 \mathrm{cc}$ and the Urethral D30 was $54.52 \%$.

He returned on October 12, 2011 for his first post-treatment follow up examination. On presentation he described nocturia of $0-1$ time per night and a daytime voiding interval of 1-2 hours. He was having moderate urinary urgency and a weak urinary stream with associated straining, but denied any incomplete bladder emptying, dysuria, hematuria or urinary incontinence. He further denied any GI complaints and stated that his erections were firm for intercourse without medication. His self-assessed American Urological Association (AUA) prostate symptom score was 21 (pre-salvage AUA symptom score 5) and his Sexual Health Inventory for Men (SHIM) score was 22. He was sent for repeat multi-parametric prostate MRI on October 25, 2011 (Fig. 2). The images showed that the previously visualized lesion in the left mid gland of the peripheral zone with rapid contrast wash in and wash out, and diffusion restriction, was no longer visualized. This was consistent with a response to treatment.

His first post treatment PSA on December 12, 2011 was $0.80 \mathrm{ng} / \mathrm{dl}$. Most recently on June 14, 2012 it had decreased further to $0.52 \mathrm{ng} / \mathrm{dl}$. He is currently without clinical evidence of disease and has returned to his pre-treatment functional baseline with regard to GU and GI symptoms.

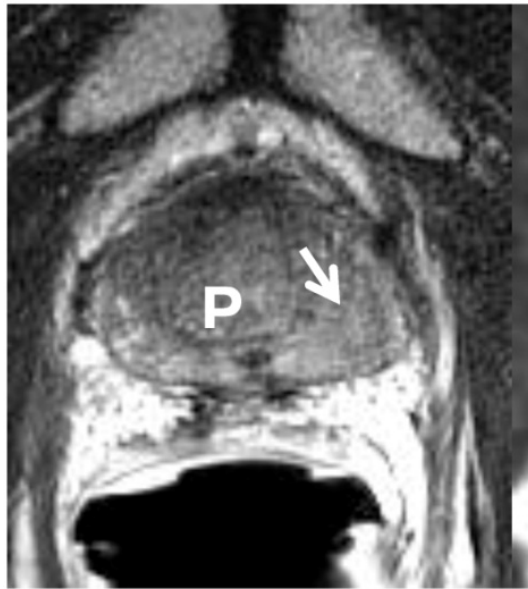

A

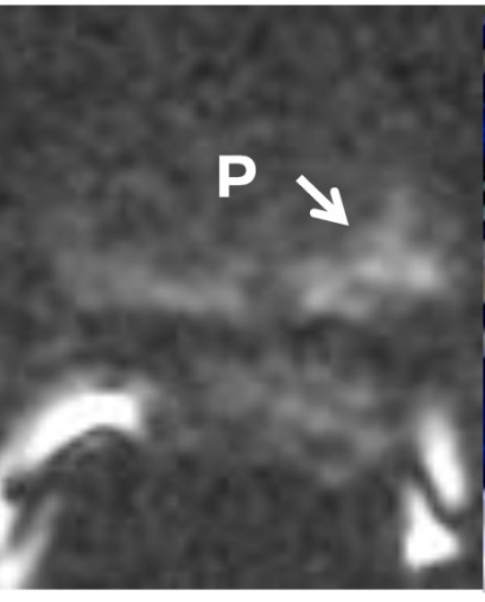

$\mathrm{B}$

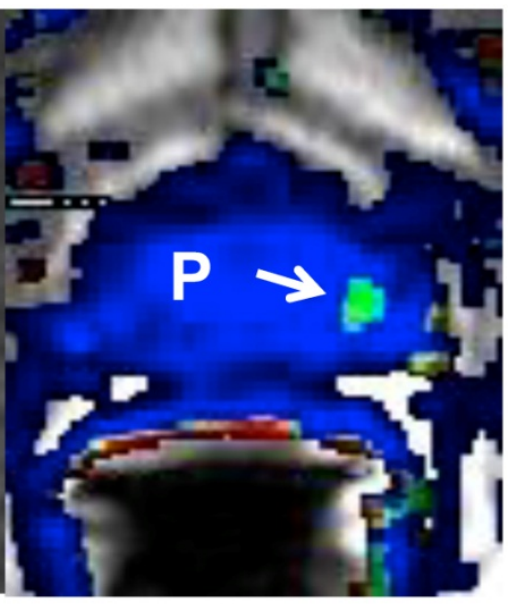

C

Figure I. Multiparametric Prostate MRI demonstrating recurrent prostate cancer at the left mid peripheral zone. (A) Axial T2 weighted image shows a vague low T2 signal focus at the left mid gland of the peripheral zone (arrow) of the prostate (P). (B) Diffusion weighted image (b I000) shows a high signal focus at the left mid gland of the peripheral zone (arrow) of the prostate $(P)$ consistent with diffusion restriction. (C) Axial post dynamic contrast image shows a lesion at the left mid gland of the peripheral zone (arrow) of the prostate (P) with rapid wash in and wash out, consistent with the biopsy proven recurrent prostate cancer.

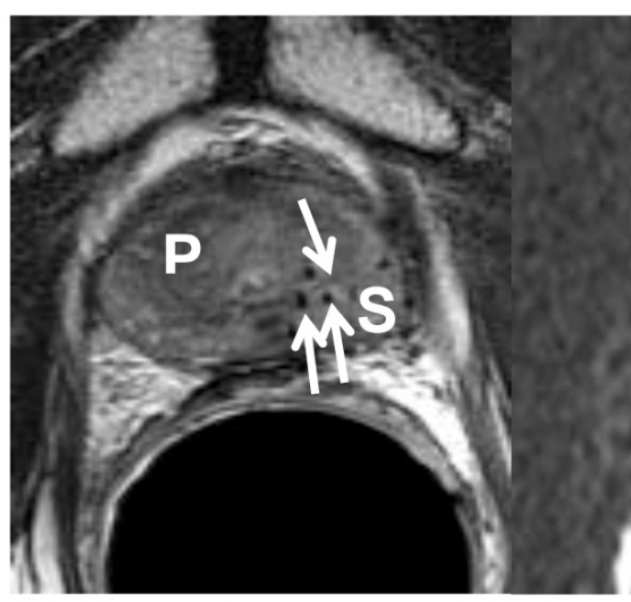

A

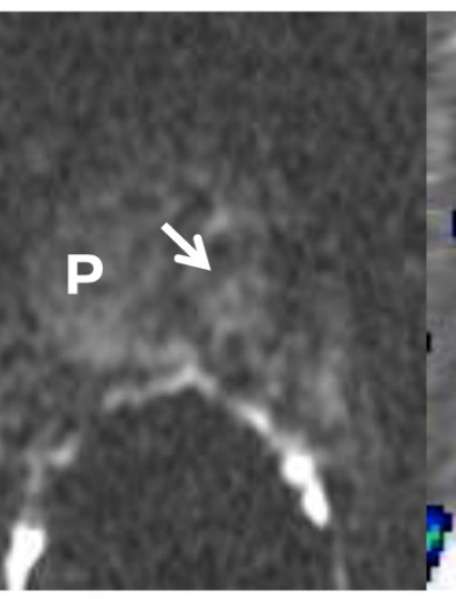

B

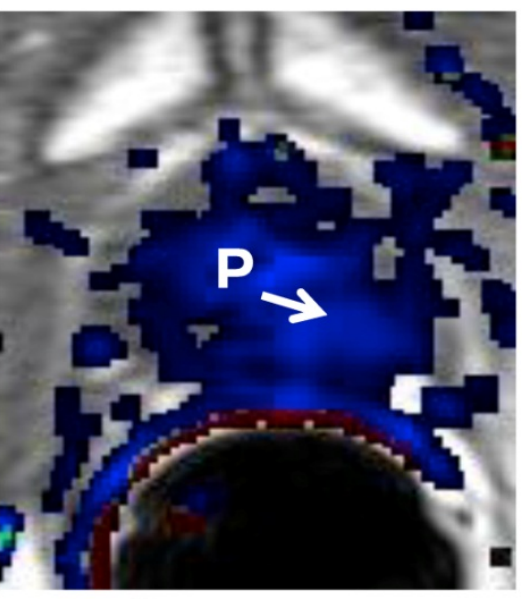

C

Figure 2. Multiparametric Prostate MRI following focal salvage brachytherapy demonstrating resolution of the recurrent prostate cancer at the left mid peripheral zone. (A) Axial T2 weighted image shows the previously visualized vague low T2 signal focus at the left mid gland of the peripheral zone of the prostate $(\mathrm{P})$ is less well seen (arrow). Multiple radiation seeds at the region $(\mathrm{S})$ are noted. (B) Diffusion weighted image (b I000) shows resolution (arrow) of the previously visualized high signal focus at the left mid gland of the peripheral zone of the prostate (P). (C) Axial post dynamic contrast image shows resolution (arrow) of the lesion at the left mid gland of the peripheral zone of the prostate $(P)$. 


\section{Discussion and state of the art}

Using the multi-parametric MRI-directed focal salvage permanent interstitial brachytherapy for locally recurrent adenocarcinoma of the prostate is a promising strategy to avoid more aggressive treatments that are associated with increased morbidity.

Today, largely due to the technological advances in image-guided radiation therapy, EBRT involves doses which are considerably greater than those used previously. Resulting from the use of radiation doses now known to be inadequate, a large number of earlier treated prostate cancers recur. Typically, the recurrence begins with a PSA rise 2-6 years after the EBRT (14). Available anatomic pathology data from salvage prostatectomy series argue that many radiation failures involve incomplete eradication of the original disease (15). It has also been reported that $>90 \%$ of all remaining pre-cancerous tissue in the irradiated prostate is found within $2 \mathrm{~mm}$ of the recurrence (16). Thus, a PSA rise which begins more than two years after EBRT may herald a focal recurrence at the original site of disease.

Even with the technological advances of dose-escalated IMRT with the addition of the latest image guidance technologies, the 10-year actuarial PSA relapse-free survival rates after primary definitive radiation therapy are between $62 \%$ and $81 \%$, depending on risk grouping (17). Differentiating between local recurrence and metastatic disease is, for obvious reasons, very important. Traditionally, bone scans and CT scans have been the tests of choice, along with physical examination, to define disease progression. Evaluation of PSA kinetics (doubling time and velocity) may be a useful tool that further aids in differentiating between local recurrence and metastatic disease. A rapidly rising PSA may indicate distant disease while a more moderate rate of PSA rise may indicate local disease recurrence (18). Although, recent data elicited debate on the use or misuse of PSA for screening and diagnosis, in the setting of radiation failure, the absolute PSA value and PSA kinetics are vital in defining treatment failure.

Traditionally, androgen suppression has been the salvage treatment of choice because of the difficulty in approach and subsequent morbidity of current salvage options. However, this form of salvage treatment is only temporizing and rarely curative secondary to the multifactorial aspects of multiple molecular pathways for the development of hormone resistance (19). Furthermore, the side effects from prolonged androgen suppression can have quality of life limiting effects. These include such as hot flashes, increased bone turnover, osteoporosis, loss of muscle mass, and increased fracture risk, sexual dysfunction, and loss of libido, memory loss, increased fat deposition, altered lipid profiles, and a significantly increased risk of cardiovascular events and cardiovascular morbidity $(20,21)$. Other salvage treatments have also proven difficult. Salvage prostatectomy, in the setting of a previously irradiated patient is challenging and its associated urinary and rectal toxicity can be severe. Traditionally, salvage cryotherapy and salvage brachytherapy have re-treated the whole prostate gland and, as such, caused high rates of treatment-related toxicity as well.

Prostate cancer imaging has advanced significantly in recent years. There is now an increased opportunity to perform prostate MRI using sequences that yield both anatomic and functional information. In addition to morphological T2-weighted sequences, the major functional techniques used for prostate imaging are diffusion-weighted MRI $(22,23)$, dynamic contrast-enhanced MRI $(24,25)$, and proton MR spectroscopic imaging (26).

Diffusion-weighted MR imaging (DW-MRI) is a technique that is sensitive to the structure of biologic tissue at the microscopic level. It is sensitive to the molecular translation of water in biologic tissues. MRI, utilizing the apparent diffusion coefficient (ADC), quantifies the effects of both diffusion and capillary perfusion. Studies using DW-MRI have shown that ADC measurements differ between prostate cancer and benign prostate tissue (27). The tumor has high cellularity, which limits the movement of water molecules within it.

Dynamic contrast-enhanced MRI (DCE-MRI) is a powerful tool in providing a functional map of blood flow and uses differences in the time course of enhancement following intravenous administration of contrast to differentiate between benign and malignant tissue. Prostate cancer often enhances more rapidly and to a higher degree with more contrast washout than the benign gland. In terms of clinical application, some studies have suggested that DCE-MRI may be of use in characterizing hypointense lesions on conventional T2 weighted MRI sequences $(28,29)$.

MR spectroscopy (MRSI) is a MRI technique that is capable of detecting and quantifying normal and cancer-related chemical compounds in the prostate gland (30). With high sensitivity $(89 \%)$ and specificity $(82 \%)$ (31), MRSI identifies 250- $\mu$ l-voxels containing prostate cancer, based on citrate, creatinine and choline chemical shift data. The technique was verified in a series of 53 prostatectomy patients with pre-operative 3D-MRSI findings upheld in greater than $90 \%$ of cases (32). What is more, by spatially re- 
solving radiation-induced atrophy, 3D-MRSI observations confirm that lower radiation doses are associated with residual metabolic activity, while permanent seed implantation produces a substantially greater level of metabolic inactivation (33). 3D-MRSI results, therefore, support the notion that brachytherapy may be an effective salvage treatment. In addition, 3D-MRSI may allow focused treatment of disease, thereby reducing treatment-related morbidity.

Recent research reported that the diagnostic accuracy of multi-parametric MR imaging in detecting recurrent prostate cancer after radiotherapy was up to $93 \%$ for those cancers with $\geq 3 \mathrm{~mm}$ biopsy core length (34). With the guidance of multi-parametric prostate MRI, salvage brachytherapy could be planned so that the radiation dose would be targeted to the area of disease recurrence while the remainder of the prostate would receive no radiation, thereby minimizing treatment-related toxicity.

\section{Conclusion}

The combination of MRI-based imaging techniques can yield quantitative information that reflects on the biological properties of prostatic tissues. These techniques provide unique information that can be used for tumor detection in the treated and untreated gland, for predicting future tumor behavior, and for monitoring the response to treatment as shown in this case. With the advent of these improved imaging modalities, it has become possible to more effectively image local recurrences within the prostate gland. This information, in conjunction with histopathological correlation, offers us a unique opportunity to safely and successfully treat recurrent prostate cancer, previously treated with definitive radiation therapy. The use of multi-parametric MRI-directed focal salvage permanent interstitial brachytherapy for locally recurrent adenocarcinoma of the prostate is a promising strategy to avoid more aggressive and expensive treatments that are associated with increased morbidity, potentially improving survival at potentially lower costs.

\section{Contributing Author Declaration}

We certify that all individuals who qualify as authors have been listed; each has participated in one or more of the following areas: conception and design of this work, the acquisition and/or analysis of data, the writing, and/or critical revision of the document, and supervision of this cooperative research effort. All contributing authors approve of the submission of this version of the manuscript and assert that the document represents valid work. If information derived from another source was used in this manu- script, we obtained all necessary approvals to use it and made appropriate acknowledgements in the document. All contributing authors take public responsibility for this work.

\section{Disclaimer}

The views expressed in this manuscript are those of the authors and do not reflect the official policy of Department of the Army, the Department of Defense or the United States Government.

\section{Copyright protection}

One of the contributing authors are military service members or employees of the U.S. Government, and this work was prepared as part of their official duties. Title 17 U.S.C. 105 provides the "Copyright protection under this title is not available for any work of the United States Government." Title 17 U.S.C. 101 defines a U.S. Government work as a work prepared by a military service member or employee of the U.S. Government as part of that person's official duties.

\section{Competing Interests}

The authors have declared that no competing interest exists.

\section{References}

1. Chade DC et al. Salvage radical prostatectomy for radiation-recurrent prostate cancer: a multi-institutional collaboration. Eur Urol. 2011 Aug; 60(2):205-10.

2. Ward JF et al. Salvage surgery for radiorecurrent prostate cancer: contemporary outcomes. J Urol. 2005 Apr; 173(4):1156-60.

3. Sanderson KM et al. Salvage radical prostatectomy: quality of life outcomes and long-term oncological control of radiorecurrent prostate cancer. J Urol. 2006 Nov; 176(5):2025-31.

4. Chade DC et al. Cancer control and functional outcomes of salvage radical prostatectomy for radiation-recurrent prostate cancer: a systematic review of the literature. Eur Urol. 2012 May; 61(5):961-71.

5. Pisters LL et al. The efficacy and complications of salvage cryotherapy of the prostate. J Urol. 1997 Mar; 157(3):921-5.

6. Grado GL et al. Salvage brachytherapy for localized prostate cancer after radiotherapy failure. Urology. 1999 Jan; 53(1):2-10.

7. Burri RJ et al. Long-term outcome and toxicity of salvage brachytherapy for local failure after initial radiotherapy for prostate cancer. Int J Radiat Oncol Biol Phys. 2010 Aug 1; 77(5):1338-44.

8. Nguyen PL et al. Magnetic resonance image-guided salvage brachytherapy after radiation in select men who initially presented with favorable-risk prostate cancer. Cancer 2007 Oct; 110(7):1485-92.

9. Rouviere $\mathrm{O}$ et al. Recurrent prostate cancer after external beam radiotherapy: value of contrast-enhanced dynamic MRI in localizing intraprostatic tumor-correlation with biopsy findings. Urology. 2004 May; 63(5):922-7.

10. Kim CK et al. Prediction of locally recurrent prostate cancer after radiation therapy: incremental value of 3T diffusion-weighted MRI. J Magn Reson Imaging. 2009 Feb; 29(2):391-7.

11. Haider MA et al. Dynamic contrast-enhanced magnetic resonance imaging for localization of recurrent prostate cancer after external beam radiotherapy. Int J Radiat Oncol Biol Phys. 2008 Feb 1; 70(2):425-30.

12. Moman MR et al. Focal salvage guided by T2-weighted and dynamic contrast-enhanced magnetic resonance imaging for prostate cancer recurrences. Int J Radiat Oncol Biol Phys. 2010 Mar 1; 76(3):741-6.

13. Hsu CC et al. Feasibility of MRimaging/MR spectroscopy-planned focal partial salvage permanent prostate implant (PPI) for localized recurrence 
after initial PPI for prostate cancer. Int J Radiat Oncol Biol Phys. 2012; [epub ahead of print].

14. Hanlon AL et al. Failure pattern implications following external beam irradiation of prostate cancer: long-term follow up and indications of cure. Cancer J. 2000 Apr; 6 Suppl 2:S193-7.

15. Arakawa A et al. High grade prostatic intraepithelial neoplasia in prostates removed following irradiation failure in the treatment of prostatic adenocarcinoma. Pathol Res Pract. 1995 Sep; 191(9):868-72.

16. Cheng $\mathrm{L}$ et al. Prevelance and distribution of prostatic intraepithelial neoplasia in salvage radical prostatectomy specimens after radiation therapy. Am J Surg Pathol. 1999 Jul; 23(7):803-8.

17. Alicikus ZA et al. Ten-year outcomes of high-dose, intensity modulated radiotherapy for localized prostate cancer. Cancer. 2011 Apr 1; 117(7):1429-37.

18. Sartor CI et al. Rate of PSA rise predicts metastatic versus local recurrence after definitive radiotherapy. Int J Radiat Oncol Biol Phys. 1997 Jul 15; 38(5):941-7.

19. Isaacs JT. The biology of hormone refractory prostate cancer. Why does it develop. Urol Clin North Am. 1999 May; 26(2):263-73.

20. Saigal CS et al. Androgen deprivation therapy increases cardiovascular morbidity in men with prostate cancer. Cancer. 2007 Oct 1; 110(7):1493-500.

21. Tsai HK et al. Androgen deprivation therapy for localized prostate cancer and the risk of cardiovascular mortality. J Natl Cancer Inst. 2007 Oct 17; 99(20):1516-24.

22. Tan $\mathrm{CH}$ et al. Diffusion weighted imaging in prostate cancer. Eur Radiol 2011; 21: 593-603.

23. Turkbey B et al. Is apparent diffusion coefficient associated with clinical risk scores for prostate cancers that are visible on 3-T MR images? Radiology 2011; 258: 488-495.

24. Alonzi R et al. Dynamic contrast enhanced MRI in prostate cancer. Eur J Radiol 2007; 63: 335-50.

25. Villers A et al. Dynamic contrast enhanced, pelvic phased array magnetic resonance imaging of localized prostate cancer for predicting tumor volume: correlation with radical prostatectomy findings. J Urol. 2006; 176:2432-37.

26. Verma $S$ et al. Prostate MRI and 3D MR spectroscopy: how we do it. AJR Am J Roentgenol 2010; 194: 1414-26.

27. Hosseinzadeh K and Schwarz SD. Endorectal diffusion-weighted imaging in prostate cancer to differentiate malignant and benign peripheral zone tissue. J Magn Reson Imaging. 2004 Oct; 20(4):654-61.

28. Preziosi $\mathrm{P}$ et al. Enhancement patterns of prostate cancer in dynamic MRI. Eur Radiol. 2003 May; 13(5):925-30.

29. Rouviere $\mathrm{O}$ et al. Characterization of time-enhancement curves of benign and malignant prostate tissue at dynamic MR imaging. Eur Radiol. 2003 May; 13(5):931-42.

30. Kurhanewicz J et al. Three-dimensional H-1 MR spectroscopic imaging of the in situ human prostate with high spatial resolution. Radiology 1996;198:795-805

31. Coakley FV et al. Endorectal MR imaging and MR spectroscopic imaging for locally recurrent prostate cancer after external beam radiation therapy: preliminary experience. Radiology. 2004 Nov; 233(2):441-8.

32. Scheidler J et al. Prostate cancer: localization with three-dimensional proton MR spectroscopic imaging - clinicopathologic study. Radiology 1999; 213:473-480.

33. Pickett B et al. Time to metabolic atrophy after permanent prostate seed implantation based on magnetic resonance spectroscopic imaging. Int J Radiat Oncol Biol Phys. 2004 Jul 1; 59(3):665-73.

34. Arumainayagam $\mathrm{N}$ et al. Accuracy of multiparametric magnetic resonance imaging in detecting recurrent prostate cancer after radiotherapy. BJU Int. 2010 Oct; 106(7):991-7. 